\title{
Head-direction coding in the hippocampal formation of birds
}

1

2

Elhanan Ben-Yishay*, Ksenia Krivoruchko*, Shaked Ron, Nachum Ulanovsky, Dori Derdikman and 3 Yoram Gutfreund

*These authors contributed equally to this work

Department of Neurobiology, Rappaport Research Institute and Faculty of Medicine, Technion, Haifa, Israel.

${ }^{¥}$ Department of Neurobiology, Weizmann Institute of Science, Rehovot, Israel.

Corresponding author: Yoram Gutfreund yoramg@technion.ac.il

\section{Abstract}

Birds strongly rely on spatial memory and navigation. Therefore, it is of utmost interest to reveal how 13 space is represented in the avian brain. Here we used tetrodes to record neurons from the 14 hippocampal formation of Japanese quails - a ground-dwelling species - while the quails roamed a 15 1x1-meter arena (>2,100 neurons from 23 birds). Whereas spatially-modulated cells (place-cells, 16 border-cells, etc.) were generally not encountered, the firing-rate of $12 \%$ of the neurons was 17 unimodally and significantly modulated by the head-azimuth - i.e. these were head-direction cells (HD cells). Typically, HD cells were maximally active at one preferred-direction and minimally at the 19 opposite null-direction, with preferred-directions spanning all $360^{\circ}$. The HD tuning was relatively 20 broad (mean width $\sim 130^{\circ}$ ), independent of the animal's position and speed, and was stable during the 21 recording-session. Similarly to findings in rodents, the HD tuning usually rotated with the rotation of a salient visual cue in the arena. These findings support the existence of an allocentric head-direction 23 representation in the quail hippocampal formation, and provide the first demonstration of head24 direction cells in birds.

\section{Introduction}

Since the seminal discovery of place cells in the rat hippocampus $[1,2]$, research on the mammalian 28 hippocampal formation has been one of the most active fields in neuroscience. Half a century of 29 extensive research resulted in a detailed characterization of hippocampal space processing in rodents, 30 and advanced the development of new techniques and paradigms for neural recording in behaving 31 animals, as well as new theories and ideas on the functional role of the hippocampus [3]. In addition to 32 place cells, a whole range of other spatial cell types have been discovered in mammals, including head- 33 
direction cells [4], spatial-view cells [5], grid cells [6], border cells [7, 8], speed cells [9], and recently 34 goal-direction cells [10]. The study of spatial processing in the hippocampus was not limited to rats but 35 expanded to other mammals, including mice, bats, monkeys and humans [11-16]. The emerging notion 36 is that the hippocampus and its related structures support spatial cognition and memory [17-19].

One important and relatively understudied question is whether the role of the hippocampal 38 formation in spatial cognition is unique to mammals, or can we find its origins in other vertebrates? 39 In this aspect, birds provide an interesting case study. Spatial navigation and foraging behaviors are 40 common in avian species, and in some cases outperform those of mammals. Migratory and homing behaviors are stunning examples of bird navigational capabilities [20-24]. In adition, the extraordinary food caching and retrieval behaviors found in some song bird species demonstrate the impressive spatial-memory capacities of birds [25-27]. What might be the neural mechanisms that support such elaborate spatial cognition in birds? And how similar are they to the hippocampal space-processing system in mammals? Answers to these questions will constitute a breakthrough in our understanding of the evolutionary origin of space processing and, through comparative studies, may contribute to understanding the underlying mechanisms and to establishing general principles of navigation and spatial memory across vertebrates.

The avian hippocampal formation (HPF), a structure in the medial dorsal cortex of the avian brain

(Fig. 1), is considered to be the homologue of the mammalian hippocampus, based on developmental, 50 topographical, genetic, and functional lines of evidence[28-32]. Growing evidence suggest that it plays 51 a role in spatial tasks $[21,24,27,28]$. For example, experiments in HPF-lesioned pigeons consistently 52 showed deficiencies in homing behaviors [33]. Similarly, lesion studies in zebra finches demonstrated that the HPF in these songbirds is involved in both learning and retention of spatial tasks [34]. 54 Investigations on the patterns of activation of immediate early genes showed enhanced activation of 55 the HPF during retrieval of cached food items [35] - a highly demanding spatial behavior - as well as 56 during maze tasks [36]. Another striking indication for the involvement of avian HPF in spatial memory 57 is found in the correlation between HPF volume and the importance of food caching for the natural 58 behavior of the species [37]. Finally, neurons with place-fields were reported in the pigeon 59 hippocampal formation, although these fields were mostly associated with rewarded locations [3860 40]. These raise the hypothesis that the role of the mammalian hippocampus in spatial cognition, and 61 the underlying neural spatial representation (place cells, grid cells, head-direction cells, etc.), have 62 their roots in earlier vertebrate evolution. However, a clear allocentric representation of space, akin 63 to that found in mammalian species, has not been reported yet in either the avian hippocampus, or in 64 any other non-mammalian vertebrate. 
In this study we report results of single unit recordings in the hippocampal formation of Japanese 67 quails (Coturnix japonica). The Japanese quail is a ground-dwelling foraging bird, which has been 68 extensively used as an animal model in developmental biology [41]. However, to our knowledge, single 69 unit recordings in behaving quails have not been done before. The natural ground-foraging behavior of 70 quails in relatively small areas is reminiscent of the rodents' small-scale foraging behavior. We therefore 71 asked if the resemblance of foraging behavior between quails and rodents implies resemblance in spatial 72 representation. To this end, we used tetrode microdrives to perform electrophysiological recordings in 73 freely behaving quails that explored a square arena, while we tracked their position and head-direction. 74 Analysis of more than 2000 putative single-units in the HPF did not reveal clear spatially-modulated cells 75 (e.g. place cells). However, we found that about $12 \%$ of the recorded cells showed a statistically 76 significant (yet broad) head-direction response, which maintained stability over time, space, and across 77 speeds. We thus provide the first evidence for a head-direction system in an avian species, shedding 78 new light on the evolutionary and functional homology of the hippocampal system across taxa.

No evidence for rodent-like place cells in the hippocampal formation of quails

We recorded and isolated 2,316 single units from 23 quails. In several of the quails, the signal-tonoise levels of the units were substantially reduced in the days following the surgery - hence the large variation in the number of cells collected from each quail - from 4 cells in quail 30 to 514 cells in quail 24 (Supplementary Table 1). Tetrodes were implanted in the left hemisphere in 21 out of the 23 quails and were stereotactically targeted to a zone within $5 \mathrm{~mm}$ rostrally from the cerebellar rostral tip, and 86 within $2 \mathrm{~mm}$ laterally to the midline (Fig. 1a-b). 19 Penetration locations were reconstructed post87 mortem by measuring the rostral and lateral distances of the penetration sites from the rostral tip of 88 the cerebellum (Fig. 1b). In 4 quails the tetrode track was additionally reconstructed with an 89 electrolytic lesion (Fig. 1c). In two of the birds penetration locations were reconstructed from microCT 90 scans (Fig. 1a). Across the recorded population, firing rates and spike widths varied; but we could not detect systematic clustering of cell spike types (Fig. 1d) nor systematic variation of firing-rates along 92 the rostro-caudal axis (Fig. 1e).

During the experiments, the quails sometimes explored the arena relatively evenly and sometimes 94 in a restricted manner (Fig. 2a and Supplementary Fig. 1). In many cases, such as the example cell 95 shown in Figure 2, the firing rate as a function of the quail's position (rate map) displayed broad spatial firing across the arena (Fig. 2b). In this example cell, spatial information was not statistically significant 97 (spatial information was smaller than the $99^{\text {th }}$ percentile of the shuffles). Only 19 cells (out of 938 cells 98 that were recorded in behavioral sessions where the quail covered more than $50 \%$ of the arena) 
passed the criteria for place cells (spatial information larger than the $99^{\text {th }}$ percentile of the shuffles $[p<0.01])$. These neurons mostly did not display a clear single field of activity in their rate-maps study.

We next moved to analyzing the relationship between the firing rate and the head direction. In the example cell, firing rate was clearly modulated by the head direction (Fig. 2c), rising from $2 \mathrm{~Hz}$ at $180^{\circ}$ 106 azimuth to more than $6 \mathrm{~Hz}$ at $10^{\circ}$ azimuth (Fig. 2d). The Rayleigh vector length (see Methods; termed thereafter 'Rayleigh vector') of the cell was 0.217 ; when compared to 1000 shuffled spike-trains, the 108 observed Rayleigh vector was well above the $99^{\text {th }}$ percentile of the shuffled distribution (Fig. 2e) - and 109 thus we categorized this example cell as having a significant HD response $(p<0.01)$. The preferred direction of the response (computed as the direction of the Rayleigh vector) pointed roughly NorthEast (red bar in Fig. 2d).

Out of the recorded population, 260 cells ( 12\%) passed the shuffling statistical test as head direction cells (with a chance level of 1\%; Supplementary Table 1). To confirm that the number of HD cells is not biased by the parameters used to define a single-unit (isolation distance and L-ratio criteria; see Methods), we analyzed the percentage of HD cells at 8 different combinations of criteria (Supplementary Table 2). The value of roughly $12 \%$ HD cells was maintained regardless of the combination of criteria used.

\section{Stability and distribution of preferred directions}

In general, the HD modulation tended to be relatively broad (Fig. 3a). The mean tuning-width (width at half height, measured halfway between maximum and minimum of the tuning) for the population of significant HD cells was $130.4^{\circ} \pm 54^{\circ}$ (Fig. $3 \mathrm{~b}$; mean \pm s.d.). Moreover, the modulation depth (normalized difference between the highest and lowest firing rate) varied between 0.4 and 1 , with a mean of 0.66 (Fig. 3c). To compare these mean values with the corresponding values of head direction cells in rodents, we performed the same analyses on datasets of previously recorded neurons from the medial entorhinal cortex (MEC), parasubiculum (PaS) and the dorsal presubiculum (dPrS) of rats, areas that are well known for their robust representation of head direction (data from [42] and [43]). Out of the 2404 cells from rats that we analyzed, 1046 were significant HD cells, comprising $44 \%$ (compared to $12 \%$ in the quails' HPF). The distribution of widths at half height, the standard deviations of the rate curves, and the Rayleigh vectors of HD cells - all pointed to substantially narrower tuning of rat HD cells as compared to quail HD cells (Supplementary Fig. 3). 
Moreover, the mean modulation depth of the HD cells in rats was substantially larger compared to the HD cells in quails ( 0.85 compared with 0.64 in quails; Supplementary Fig. 3 ).

Despite their relatively broad shape, the HD tuning curves in the quails had high directional stability: (i) The preferred directions in the first and second halves of the session were significantly correlated (Fig. 3d; Pearson correlation: $r=0.95, p<0.0001, n=186$; the numbers of neurons in this and following comparisons is lower than $n=260$ because we required sufficient coverage in both halves: see Methods). (ii) The preferred directions computed from the north versus south halves of the arena were significantly correlated (Fig. 3e; Pearson correlation: $r=0.88, p<0.0001, n=175$ ), and likewise for preferred directions from the west versus east halves (Fig. 3f; Pearson correlation: $r=0.90, p<0.0001$, $n=169$ ). (iii) Because some of the quails mostly explored the borders of the arena (see examples in Supplementary Fig. 1), we also analyzed the preferred head directions when the quails were near the border versus when they were in the center of the arena; these were also found to be highly significantly correlated (Fig. 3g; Pearson correlation: $r=0.88, p<0.0001, n=122$ ). (iv) Finally, the preferred directions during fast movement of the quail $(>5 \mathrm{~cm} / \mathrm{sec}$ ) were significantly correlated with the preferred directions during slow movements ( $<5 \mathrm{~cm} / \mathrm{sec}$ ) (Fig. 3h; Pearson correlation: $r=0.97$, $p<0.0001, n=260$ ). In all these tests the regression line was very close to the $45^{\circ}$ line (Fig. $3 d-h$ : compare red and black lines). Our results indicate a stable head-direction representation over time and space, as well as over the speed of the quails. The significant correlation between the preferred 149 directions in different parts of the arena argue against the possibility that the head-direction tuning is an outcome of spatial view cells [5].

In rodents, HD cells commonly rotate their preferred direction following rotation of salient cues in the arena $[4,44]$. We therefore positioned, in some of the experiments, two LED light-sources in 153 opposite sides of the arena, have lit only one of them, and then switched the side of the illuminating source at mid-session. 52 significant HD cells were analyzed in this experiment. Following the switch, 155 some of the neurons demonstrated clear $180^{\circ}$ rotation of their tuning curve (Fig. 3I). At the population 156 level, the distribution of the preferred-direction differences between light on one side and light on the 157 $180^{\circ}$-opposite side was centered near $180^{\circ}$ (Fig. $3 \mathrm{~J}$ and $3 \mathrm{~K}$; mean angle \pm circular standard deviation $=$ 158 $\left.162 \pm 64^{\circ}\right)$, and significantly deviated from the distribution of the mean directional shift between the 159 two halves of the session without any cue-changes (Fig. 3J and 3K; Watson-Williams mean angle test, 160 $p<0.0001)$.

The preferred directions of the population covered almost uniformly the entire range of $360^{\circ}$ (Fig. 4a). This uniformity was seen when pooling data from all quails (Fig. 4a), as well as in most of the 163 individual quails (Supplementary Fig. 4). Significant HD cells, spanning all possible directions, have 
been recorded at all the recording-depths in the HPF and at all anatomical locations within HPF (Fig.

$4 b-c)$. HD cells were found at nearly all depths from the brain-surface, and all distances rostral to the 166 cerebellum tip (Fig. 4d-e). Thus, our results do not indicate a clear tendency for anatomical clustering of HD cells or anatomical clustering of best directions. We conclude that significant and stable HD cells are found throughout the HPF of quails.

\section{Discussion}

To our knowledge, this is the first time that a stable and abundant population of head-direction cells is reported in the hippocampal formation of an avian species. Neurons that represent head direction in allocentric coordinates during real-world movements have been reported in mammals [4, 45-47], and very recently in fish [48] - as well as in head-fixed insects in virtual reality [49, 50]. These cells are thought to underlie the directional sense of animals, which is vitally important for goal directed navigation and other spatially coordinated behaviors [51]. Because many species of birds display remarkable cognitive capabilities that are related to spatial behaviors and spatial memory [20direction is that they exhibit a uniform and stable representation of directions [51, 52]. Moreover, direction sensitivity is maintained independent of the speed of the animal [4]. Here we report a population of neurons that are modulated by the head direction of the quail: these neurons uniformly

Networks of head-direction cells in mammals integrate angular velocity information coming from sub-division homology between the avian HPF and the mammalian HPF, is not well established [28]. Atoji and Wild $(2004,2006)$ provided a histochemical division of the pigeon HPF to dorsal lateral (DL), dorsal medial (DM) and a medial V shape complex $[30,58]$. The homology of this subdivision with the mammalian HPF is debated, with the controversy focused mostly around the homologies of the Vshaped complex and the dorsal medial parts with the mammalian Dentate Gyrus and Ammon's horn 
were in the more accessible dorsal lateral (DL) and dorsal medial (DM) parts of the HPF (Fig. 1b and

Fig. 4b) - thus, our findings possibly represent an avian head-direction system homologous to the connections from visual Wulst [67] as well as connections from the dorsal thalamus [68], which may provide the anatomical substrates for integration of visual and self-motion cues. Yet, obvious of population of neurons recorded from rats in the Pas, dPrS and MEC - using the exact same analysispipeline - and have confirmed the substantial differences in modulation depth, tuning broadness and abundance of HD cells (Supplementary Fig. 3). The relatively low abundance of HD cells in our findings may reflect the non-focal sampling of neurons in our study: Perhaps a higher fraction of HD cells would be found in specific sub regions of the HPF. However, our results showed no evidence for anatomical

Functional hemispheric lateralization is common in birds with laterally-placed eyes [71]. Recordings in the pigeon HPF demonstrated a significant difference in the behavior of neurons between the two hemispheres: neurons in the left HPF tended to code spatial parameters more reliably, while neurons in the right HPF tended to code goal locations [40]. Lateralization of HPF spatial coding is also supported by a lesion study in pigeons showing that an intact left but not right HPF is required for (quails \# 10 and 23; Supplementary table 1) and found 11 HD cells out of 123 cells (9\%). Thus, we have no indication for lateralized organization of HD cells in the quail. However, further sampling is required for a thorough comparison between hemispheres. 
Another striking difference between neural responses in rodents and the results reported here is spatial modulation of their firing rates, but these were mostly broadly tuned (Supplementary Fig. 2). relatively broad spatial tuning [73]. On the other hand, behavioral experiments in quails [74], pigeons [75] and zebra finches [76] strongly support well-developed spatial cognitive abilities in these bird species - such as novel shortcuts, model-based spatial learning, navigation, and spatial memory. It is possible that the neural substrate of spatial perception of birds is not localized in the HPF, or alternatively, it is localized in a specific sub-region of the HPF which has not been explored yet. However, lesion studies in quails, pigeons and zebra finches $[34,77,78]$, as well as gene expression analysis [79, 80], provide strong evidence that the HPF is critically important for spatial cognition. A different possibility is that the HPF of both mammals and birds holds the neural basis of spatial cognition but evolved differently: rather than having a large population of highly-specific and sparsely encoding cells - as seen in mammals - birds might possess a population-coding scheme which is carried by a population of neurons with broad tuning and non-uniform fields. This possibility is consistent with our findings of head direction tuning which was broadly tuned.

A preliminary study in food caching birds that are specialized in spatial memory (tufted titmouses) revealed place cells in the rostral HPF [73], in the same areas that have been explored in other birds, including here in quails - but without observing place cells. Taken together, our and previous findings suggest the notion that the HPF in birds generally supports spatial cognition - but that the detailed coding scheme is species-specific and evolved differently according to the ecological needs of the species. Previous studies of hippocampus activity in freely behaving pigeons did not track the headdirection[39] and therefore it is not known if HD cells can be found in other species of birds. However, in the above-mentioned preliminary study of a food caching species, head direction was tracked, and HD cells were observed in addition to place cells [73]. Our study is thus unique to report HD cells in an avian species lacking place-cells. This is an interesting observation and taken together with observations of HD cells in a variety of species - ranging from insects, fish and birds - it suggests that HD cells, and not place cells, were conserved through evolution. These intriguing inter-species 
linking it to the animals' behavioral repertoire and evolutionary history: A merging of neuroscience,

Animals

Japanese quails (Cotrunix japonica) of both sexes were used in this study. The quails were hatched

and raised in our in-house breeding colony, housed in $1 \times 1 \mathrm{~m}$ cages and maintained on a 12/12hr

Adult Japanese quails (females and males; weight: $150-250$ g, age: 4-12 months old) were prepared for repeated electrophysiological recordings with a single surgical procedure: the birds were positioned in a stereotaxic frame (Kopf, small animals instrument Model 963) using Kopf rat ear bars.

Head angle was controlled with a biting bar that was positioned $45^{\circ}$ below the inter-aural axes (resembling the standard position in the pigeon and the quail atlases $[81,82)$. At this head position, in 
Electrophysiological recordings were conducted in a $1 \times 1 \mathrm{~m}$ open-field arena. The birds were released

to roam spontaneously in the arena. Occasionally, food items were scattered in the arena to

encourage movement. A tethered 16-channel headstage (Intan RHD2132) was attached to the drive

Electrical recording was sampled at $30 \mathrm{kHz}$ using the Cheetah 6.0 software (Neuralynx), which records

During subsequent processing (off-line), the electrical recordings were filtered between $600-6,000$ 


$$
\operatorname{Thr}=4 * \text { median }\left\{\frac{|x|}{0.6745}\right\}
$$

Where $x$ is the filtered recorded signal, the division by 0.6745 accounts for the relationship between 330 the median of the noise and the standard deviation of a normal distribution. Voltage threshold was calculated over 1-minute recording windows. Electrical artifacts were detected and removed from all channels based on absolute voltage. Following artifact removal, a spike detection algorithm was used for each tetrode: whenever one channel crossed the threshold value, a 1-ms wide segment was saved from all channels on the tetrode, where the peak was centered over the $8^{\text {th }}$ sample (for a total of 32 samples). Coincidence detection algorithm was used to further identify and remove movement 336 artifacts; large-amplitude events occurred within all channels in each tetrode or between tetrodes were removed from analysis (Supplementary Fig. 5b). Additionally, all detected spikes were compared 338 to a pre-existing spike-shape database (template matching) by correlating different segments of the 339 spike shape to each template from the database. A spike was included for further analysis if its Pearson-correlation coefficient with any of the spike templates was larger than 0.8.

Manual spike sorting was performed offline using SpikeSort3D software (Neuralynx) and consisted of plotting the spikes in 3D parameter space, finding the features which give the best cluster separation and performing manual cluster cutting. Clustering quality was assessed based on two measures: isolation distance and $L_{\text {ratio }}$ [84]. These parameters estimate how well-separated is the cluster of spikes from the other spikes and noise recorded on the same tetrode. A well-separated cluster has a large isolation distance and a small $\mathrm{L}_{\mathrm{ratio}}$. A cluster was considered a single unit if it had an $\mathrm{L}_{\text {ratio }}$ smaller than 0.2 and an isolation distance larger than 15 . When the number of points outside the isolated cluster is smaller than half of the total points in the tetrode it is not possible to obtain the isolation distance value ( $\mathrm{NaN}$ isolation distance). Clusters with $\mathrm{NaN}$ isolation distance were included in the analysis (see 350 Supplementary Table 2). Additionally, clusters were categorized as multi- or single-units based on their inter-spike interval histogram: a cluster was categorized as single-unit if less than $5 \%$ of its spikes occurred less than $2 \mathrm{~ms}$ after the previous spike.

\section{Histology}

In a few of the quails, an electrical lesion was performed by injecting a positive current through one 355 of the tetrodes $(+5 \mu \mathrm{A}$ for $20 \mathrm{sec})$. A week later, the quail was deeply anesthetized and perfused with phosphate buffer solution (PBS) followed by $4 \%$ paraformaldehyde. The brain was removed and stored in $4 \%$ paraformaldehyde for $2-3$ days at $4^{\circ} \mathrm{C}$, then transferred to PBS. Following fixation, the quail 358 brains were dehydrated in 70\%, 80\%, 95\% and 100\% ethanol, cleared in Xylene and embedded in 359 paraffin wax. The paraffin-embedded brains were coronal sectioned at $5 \mu \mathrm{m}$ using a microtome (RM 360 2265 Leica). Sections collected at $40 \mu \mathrm{m}$ intervals were mounted on superfrost glass slides and dried 
in an oven at $37^{\circ} \mathrm{C}$ for 24 hours. After drying the sections, they were deparaffinized in xylene,

\section{Data analysis}

Single units were included for further analysis only if at least 300 spikes occurred during the session,

Spatial firing-rate maps were computed by partitioning the arena into $3 \times 3 \mathrm{~cm}$ bins. The number of

spikes in each bin was divided by the time the bird spent in that bin. Bins which the quail visited for

$$
I=\sum_{x} \frac{p_{x} \lambda_{x} \log _{2}\left(\frac{\lambda_{x}}{\lambda}\right)}{\lambda}
$$

Where $\lambda$ is the mean firing rate of the cell, $\lambda_{x}$ is the mean firing rate in bin $x$ ( $x$ includes only bins that the quail visited), $p_{x}$ is the probability of occupying bin $x$. Spatial correlation was the 2-dimensional correlation coefficient between the rate map of the first and second halves of the session - an index of stability. For statistical evaluation of the spatial modulation, a shuffling procedure was applied. The entire spike train was rigidly and circularly shifted by a random interval (intervals smaller than $\pm 20 \mathrm{~s}$ were not used). This was repeated 1000 times for each neuron, and for each of the shuffles the spatial information index was calculated. The experimentally observed index for the actual neuron was considered statistically significant with a P value larger than 0.01 if it surpassed $99 \%$ of the shuffle indices. For display purposes, the rate maps were smoothed using a 2D Gaussian kernel ( $\sigma=1$ bins), but all the computations and indexes were calculated without smoothing.

Head-direction was computed as the perpendicular orientation from the line connecting the positions

$$
R=\frac{\left|\sum w \times e^{i \alpha}\right|}{\sum w}
$$


Where $w$ is the firing-rate per bin and $\alpha$ is the bin direction. The preferred (mean) direction of the 393 tuning curve was estimated by the direction of the Rayleigh vector:

$$
\text { preferred direction }=\operatorname{Arg}\left(\sum w \times e^{i \alpha}\right)
$$

The statistical significance of the directionality was assessed by applying a spike-shuffling procedure as described above. Shuffling was repeated 1000 times, and for each shuffle the Rayleigh vector (RV) length was calculated. A neuron was considered head-direction modulated if the RV score was larger 398 than $99 \%$ of the shuffled scores. A shuffling procedure was used only for tuning curves with a RV score 399 higher than 0.10 .

The stability of the HD preferred direction was assessed by dividing the time of the experiment into 401 two halves (Fig. 3d); by dividing the arena into two regions: south half versus north half, east half 402 versus west half, and center region versus border region (Fig. 3e-g); and by dividing the quail's speed 403 to two speeds: below $5 \mathrm{~cm} / \mathrm{sec}$ and above $5 \mathrm{~cm} / \mathrm{sec}$ (Fig. 3h). Preferred HD was calculated separately 404 for each part. This analysis was performed only for the sessions where the head direction coverage 405 was larger than $50 \%$ in both regions. Head direction coverage was calculated by dividing the time spent at each region by 60 (the number of $6^{\circ} \mathrm{HD}$ bins) and thus obtaining the expected time at each 406 direction-bin if the behavioral head directions were uniformly distributed. The percent coverage was then defined as the percentage of directional bins in which the quail spent more than $75 \%$ of the 408 expected uniform time (see Supplementary Fig. 1 for examples).

\section{Acknowledgments}

We thank Dr. Yael Zahar for assistance and support, and May-Britt Moser, Edvard I. Moser and

Charlotte N. Boccara for usage of rat data in Supplementary Fig. 3. This work was supported by research grants from the Rappaport Institute for Biomedical Research, the Adelis Foundation and the Israel Science Foundation. Yoram Gutfreund also acknowledges the generous support of the Edward

\section{Author contributions}

E.B. ,K.K. and S.R. performed the experiments, wrote code, analyzed data and produced graphs and 
1. O'Keefe, J., and Dostrovsky, J. (1971). The hippocampus as a spatial map. Preliminary evidence

2. O'Keefe, J., and Nadel, L. (1978). The hippocampus as a cognitive map, (Oxford; New York:

3. Eichenbaum, H. (2017). The role of the hippocampus in navigation is memory. J Neurophysiol $117,1785-1796$

4. Taube, J.S., Muller, R.U., and Ranck, J.B. (1990). Head-direction cells recorded from the 420-435.

5. Rolls, E.T. (1999). Spatial view cells and the representation of place in the primate hippocampus. Hippocampus 9, 467-480.

6. Hafting, T., Fyhn, M., Molden, S., Moser, M.B., and Moser, E.I. (2005). Microstructure of a spatial map in the entorhinal cortex. Nature 436, 801-806.

7. Solstad, T., Boccara, C.N., Kropff, E., Moser, M.B., and Moser, E.I. (2008). Representation of geometric borders in the entorhinal cortex. Science 322, 1865-1868.

8. Lever, C., Burton, S., Jeewajee, A., O'Keefe, J., and Burgess, N. (2009). Boundary vector cells in

9. Kropff, E., Carmichael, J.E., Moser, M.B., and Moser, E.I. (2015). Speed cells in the medial entorhinal cortex. Nature 523, 419-424.

10. Sarel, A., Finkelstein, A., Las, L., and Ulanovsky, N. (2017). Vectorial representation of spatial goals in the hippocampus of bats. Science $355,176-180$.

11. McHugh, T.J., Blum, K.I., Tsien, J.Z., Tonegawa, S., and Wilson, M.A. (1996). Impaired 1349.

12. Ulanovsky, N., and Moss, C.F. (2007). Hippocampal cellular and network activity in freely

13. Yartsev, M.M., Witter, M.P., and Ulanovsky, N. (2011). Grid cells without theta oscillations in

14. Killian, N.J., Jutras, M.J., and Buffalo, E.A. (2012). A map of visual space in the primate

15. Ono, T., Nakamura, K., Nishijo, H., and Eifuku, S. (1993). Monkey hippocampal neurons related

16. Ekstrom, A.D., Kahana, M.J., Caplan, J.B., Fields, T.A., Isham, E.A., Newman, E.L., and Fried, I. 455 (2003). Cellular networks underlying human spatial navigation. Nature 425, 184-188. 
18. Anderson, M.I., Killing, S., Morris, C., O'Donoghue, A., Onyiagha, D., Stevenson, R., Verriotis, M., and Jeffery, K.J. (2006). Behavioral correlates of the distributed coding of spatial context. Hippocampus 16, 730-742.

19. Geva-Sagiv, M., Las, L., Yovel, Y., and Ulanovsky, N. (2015). Spatial cognition in bats and rats:

20. Wiltschko, W., and Wiltschko, R. (2012). Global navigation in migratory birds: tracks, strategies, and interactions between mechanisms. Curr Opin Neurobiol 22, 328-335.

21. Mouritsen, H., Heyers, D., and Gunturkun, O. (2015). The Neural Basis of Long-Distance Navigation in Birds. Annu Rev Physiol.

22. Thorup, K., Bisson, I.-A., Bowlin, M.S., Holland, R.A., Wingfield, J.C., Ramenofsky, M., and 468 Wikelski, M. (2007). Evidence for a navigational map stretching across the continental US in a $\quad 469$ migratory songbird. Proceedings of the National Academy of Sciences 104, 18115-18119. 470

23. Wallraff, H.G., and Wallraff, H.G. (2005). Avian navigation: pigeon homing as a paradigm, (Springer Science \& Business Media).

24. Bingman, V.P., Hough, G.E., 2nd, Kahn, M.C., and Siegel, J.J. (2003). The homing pigeon

25. Grodzinski, U., and Clayton, N.S. (2010). Problems faced by food-caching corvids and the

26. Healy, S.D., and Hurly, T.A. (2004). Spatial learning and memory in birds. Brain Behav Evol 63, 211-220.

27. Healy, S.D., de Kort, S.R., and Clayton, N.S. (2005). The hippocampus, spatial memory and food hoarding: a puzzle revisited. Trends in ecology \& evolution 20, 17-22.

28. Herold, C., Coppola, V.J., and Bingman, V.P. (2015). The maturation of research into the avian

29. Karten, H.J. (1969). Organization of Avian Telencephalon and Some Speculations on Phylogeny

30. Atoji, Y., and Wild, J.M. (2006). Anatomy of the avian hippocampal formation. Rev Neurosci 17, 3-15.

31. Chen, C.C., Winkler, C.M., Pfenning, A.R., and Jarvis, E.D. (2013). Molecular profiling of the

32. Colombo, M., and Broadbent, N. (2000). Is the avian hippocampus a functional homologue of the mammalian hippocampus? Neurosci Biobehav Rev 24, 465-484.

33. Gagliardo, A., loale, P., and Bingman, V.P. (1999). Homing in pigeons: the role of the 494 hippocampal formation in the representation of landmarks used for navigation. J Neurosci 19, 311-315. 
35. Smulders, T.V., and DeVoogd, T.J. (2000). Expression of immediate early genes in the hippocampal formation of the black-capped chickadee (Poecile atricapillus) during a foodhoarding task. Behav Brain Res 114, 39-49.

36. Mayer, U., and Bischof, H.J. (2012). Brain activation pattern depends on the strategy chosen by zebra finches to solve an orientation task. J Exp Biol 215, 426-434.

37. Pravosudov, V.V., and Smulders, T.V. (2010). Integrating ecology, psychology and neurobiology

38. Hough, G.E., and Bingman, V.P. (2004). Spatial response properties of homing pigeon

39. Kahn, M.C., Siegel, J.J., Jechura, T.J., and Bingman, V.P. (2008). Response properties of avian 191, 153-163.

40. Siegel, J.J., Nitz, D., and Bingman, V.P. (2006). Lateralized functional components of spatial cognition in the avian hippocampal formation: evidence from single-unit recordings in freely moving homing pigeons. Hippocampus 16, 125-140.

41. Poynter, G., Huss, D., and Lansford, R. (2009). Japanese quail: an efficient animal model for the

42. Gofman, X., Tocker, G., Weiss, S., Boccara, C.N., Lu, L., Moser, M.B., Moser, E.I., Morris, G., and

43. Boccara, C.N., Sargolini, F., Thoresen, V.H., Solstad, T., Witter, M.P., Moser, E.I., and Moser,

44. Dudchenko, P.A., Goodridge, J.P., and Taube, J.S. (1997). The effects of disorientation on visual

45. Finkelstein, A., Derdikman, D., Rubin, A., Foerster, J.N., Las, L., and Ulanovsky, N. (2015).

46. Taube, J.S., and Muller, R.U. (1998). Comparisons of head direction cell activity in the

47. Robertson, R.G., Rolls, E.T., Georges-François, P., and Panzeri, S. (1999). Head direction cells in the primate pre-subiculum. Hippocampus 9, 206-219.

48. Vinepinsky, E., Cohen, L., Perchik, S., Ben-Shahar, O., Donchin, O., and Segev, R. (2020).

49. Seelig, J.D., and Jayaraman, V. (2015). Neural dynamics for landmark orientation and angular path integration. Nature 521, 186-191.

50. Turner-Evans, D.B., and Jayaraman, V. (2016). The insect central complex. Current Biology 26, 
51. Munn, R.G., and Giocomo, L.M. (2020). Multiple head direction signals within entorhinal cortex: origin and function. Current Opinion in Neurobiology 64, 32-40.

52. Cullen, K.E., and Taube, J.S. (2017). Our sense of direction: progress, controversies and challenges. Nature Neuroscience 20, 1465.

53. Hulse, B.K., and Jayaraman, V. (2019). Mechanisms Underlying the Neural Computation of Head Direction. Annual Review of Neuroscience 43.

54. Yoder, R.M., Clark, B.J., and Taube, J.S. (2011). Origins of landmark encoding in the brain.

55. Clark, B.J., and Taube, J.S. (2012). Vestibular and attractor network basis of the head direction

56. Taube, J.S. (2007). The head direction signal: origins and sensory-motor integration. Annu.

57. Winter, S.S., Clark, B.J., and Taube, J.S. (2015). Disruption of the head direction cell network

58. Atoji, Y., and Wild, J.M. (2004). Fiber connections of the hippocampal formation and septum and subdivisions of the hippocampal formation in the pigeon as revealed by tract tracing and kainic acid lesions. J Comp Neurol 475, 426-461.

59. Krebs, J.R., Erichsen, J.T., and Bingman, V.P. (1991). The distribution of neurotransmitters and neurotransmitter-related enzymes in the dorsomedial telencephalon of the pigeon (Columba

60. Kempermann, G. (2012). New neurons for'survival of the fittest'. Nature reviews neuroscience $13,727-736$.

61. Striedter, G.F. (2016). Evolution of the hippocampus in reptiles and birds. J Comp Neurol 524, 496-517.

62. Herold, C., Bingman, V.P., Ströckens, F., Letzner, S., Sauvage, M., Palomero-Gallagher, N.,

63. Atoji, Y., Sarkar, S., and Wild, J.M. (2016). Proposed homology of the dorsomedial subdivision

64. Székely, A.D. (1999). The avian hippocampal formation: subdivisions and connectivity. 570 Behavioural brain research 98, 219-225.

66. Rattenborg, N.C., and Martinez-Gonzalez, D. (2011). A bird-brain view of episodic memory. 
67. Shanahan, M., Bingman, V.P., Shimizu, T., Wild, M., and Gunturkun, O. (2013). Large-scale

68. Trottier, C., Reperant, J., and Miceli, D. (1995). Anatomical evidence of a retino-thalamo-

69. Sargolini, F., Fyhn, M., Hafting, T., McNaughton, B.L., Witter, M.P., Moser, M.-B., and Moser,

70. Giocomo, L.M., Stensola, T., Bonnevie, T., Van Cauter, T., Moser, M.B., and Moser, E.I. (2014). 585 Topography of head direction cells in medial entorhinal cortex. Curr Biol 24, 252-262.

71. Güntürkün, O. (1997). Avian visual lateralization: a review. Neuroreport 8, iii-xi.

72. Kahn, M.C., and Bingman, V.P. (2004). Lateralization of spatial learning in the avian

73. Payne, H.L., Lynch, G.F., and Aronov, D. (2020). Precise spatial representations in the 590 hippocampus of a food-caching bird. bioRxiv, 2020.2011.2027.399444.

74. Ruploh, T., Kazek, A., and Bischof, H.J. (2011). Spatial orientation in Japanese quails (Coturnix 592 coturnix japonica). PLoS One 6, e28202.

75. Cheng, K., Spetch, M.L., Kelly, D.M., and Bingman, V.P. (2006). Small-scale spatial cognition in

76. Mayer, U., Watanabe, S., and Bischof, H.J. (2013). Spatial memory and the avian hippocampus:

77. Lormant, F., Cornilleau, F., Constantin, P., Meurisse, M., Lansade, L., Leterrier, C., Lévy, F., and science.

78. Bingman, V.P., and Yates, G. (1992). Hippocampal lesions impair navigational learning in

79. Mayer, U., Watanabe, S., and Bischof, H.J. (2010). Hippocampal activation of immediate early

80. Sherry, D.F., Grella, S.L., Guigueno, M.F., White, D.J., and Marrone, D.F. (2017). Are There

81. Karten, H.J., and Hodos, W. (1967). A stereotaxic atlas of the pigeon brain, (Baltimore, Maryland: The Johns Hopkins Press).

82. Baylé, J. (1974). Stereotaxic topography of the brain of the quail. J Physiol Paris 68, 219-241.

83. Quiroga, R.Q., Nadasdy, Z., and Ben-Shaul, Y. (2004). Unsupervised Spike Detection and 
bioRxiv preprint doi: https://doi.org/10.1101/2020.08.31.274928; this version posted March 4, 2021. The copyright holder for this preprint (which was not certified by peer review) is the author/funder. All rights reserved. No reuse allowed without permission.

85. Skaggs, W.E., McNaughton, B.L., Wilson, M.A., and Barnes, C.A. (1996). Theta phase precession in hippocampal neuronal populations and the compression of temporal sequences. Hippocampus 6, 149-172. 

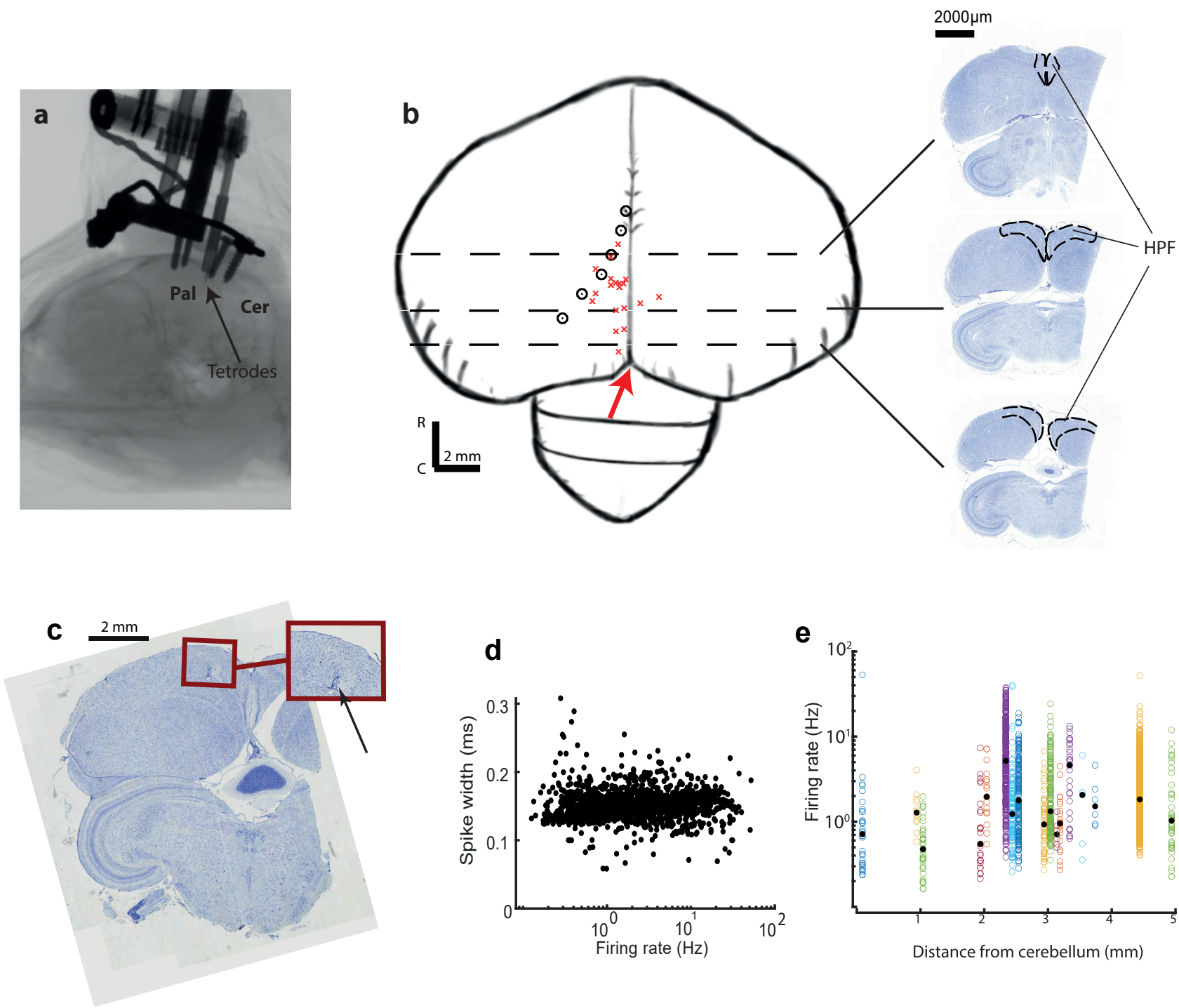

Figure 1. Recording locations and firing properties. a, Micro-CT scan showing an implanted microdrive on the quail's skull. Arrow points to the tip of the tetrodes. Pal pallium, Cer -cerebellum. b, A sketch of the quail's brain. Red X's mark the approximate recording locations from 19 quails. Black dots represent the putative lateral border of the hippocampal formation, which follows the lateral end of the lateral ventricle. Distances of black circles from the midline are approximated based on figures from the quail's brain stereotaxic atlas [82]. Dashed lines indicate the approximate coronal section levels shown on the right. The dashed lines in the insets mark the hippocampal formation (HPF). The red arrow denotes the cerebellum tip. c, A Nissl stained coronal section showing an electrolytic lesion. Inset: magnified view of the lesioned region. The arrow points to the lesion mark. d, Spike width (full width at half maximum) for all the recorded single-units as a function of their firing rate. X-axis is logarithmic. e, Firing rates of single-units versus the recording location along the rostro-caudal axis (rostral distance from cerebellum tip). Colors designate different quails. Black circles designate the median firing rates. Y-axis is logarithmic. 
bioRxiv preprint doi: https://doi.org/10.1101/2020.08.31.274928; this version posted March 4, 2021. The copyright holder for this preprint (which was not certified by peer review) is the author/funder. All rights reserved. No reuse allowed without permission.
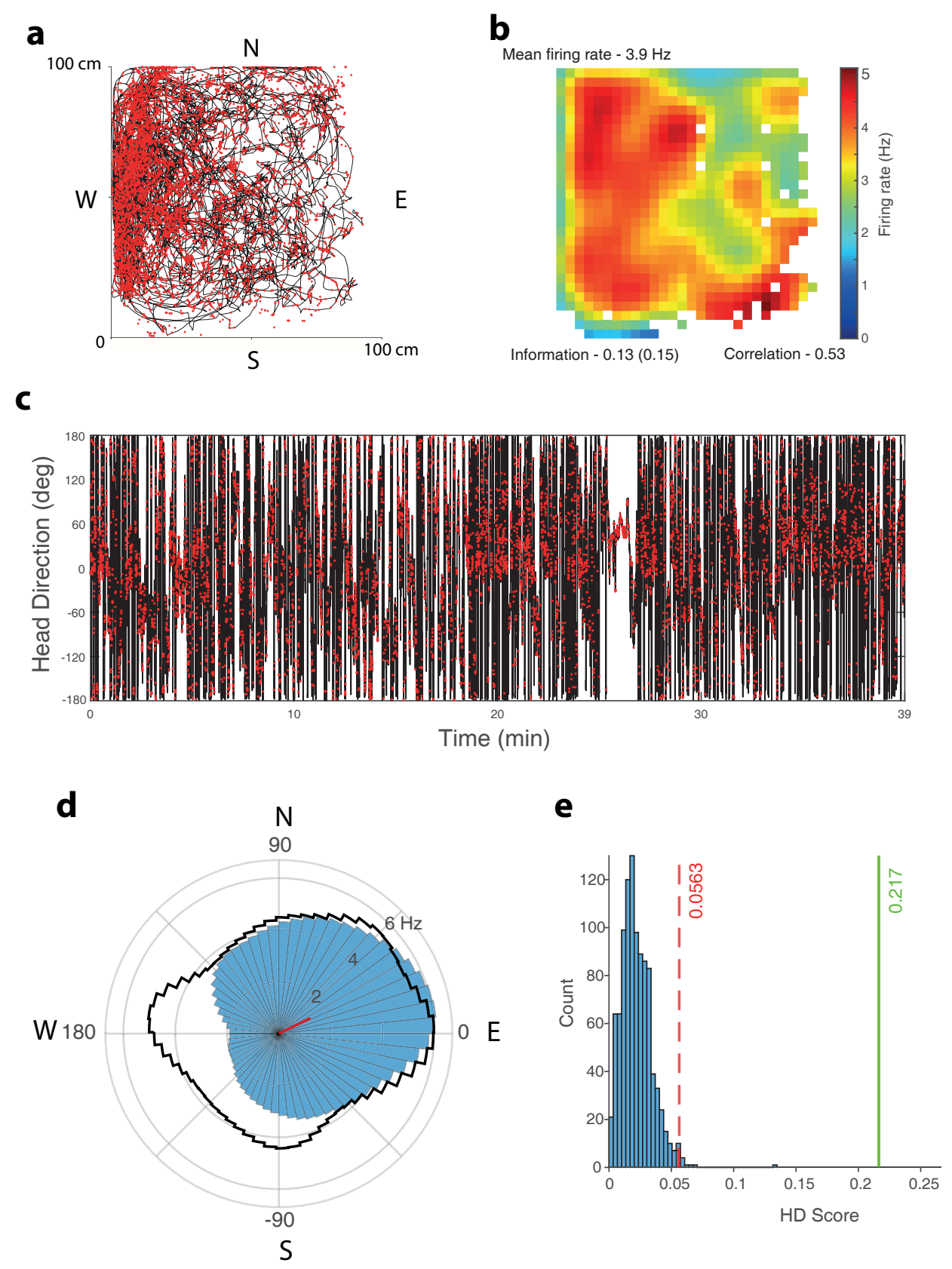

Figure 2. Data from an example neuron. a, The quail's position in the arena during a 35-min recording session is marked by a black line. Red dots are the locations of spikes fired by the neuron. $\mathrm{N}$ - north side of the arena; $\mathrm{S}$ - south, $\mathrm{E}$ - east; $\mathrm{W}$ - west. b, Firing-rate map of the neuron. Indicated are the mean firing rate (top left), spatial information in bits/spike (bottom left), and map correlation between first and second half of the session (bottom right). White pixels are pixels which were not visited at least 200 ms during the session. c, Head-direction of the quail as a function of time (black line), with spikes superimposed (red dots). d, Polar plot showing the firing rate as a function of head direction (blue). The red bar indicates the length and direction of the Rayleigh vector. The black curve shows the relative time spent behaviorally in each head-direction bin. e, Rayleigh vector shuffling histogram. Red dashed line represents the Rayleigh vector length for the $99 \%$ percentile shuffle; green line shows the observed Rayleigh vector length for this example neuron. 
a

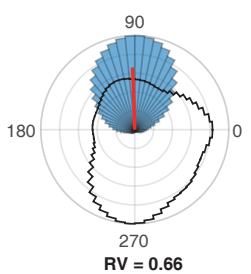

90

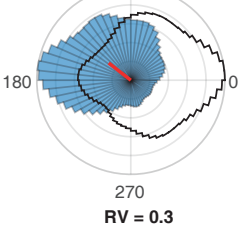

d

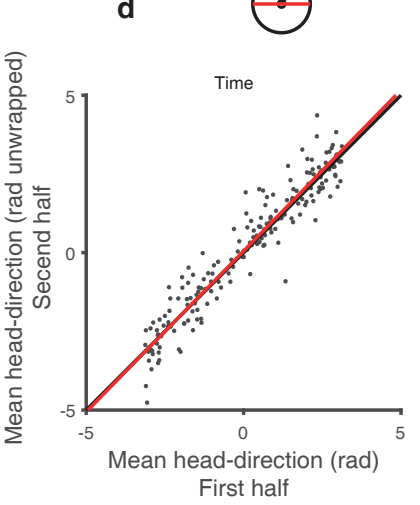

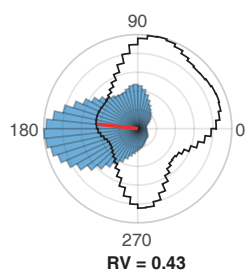

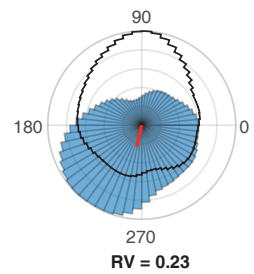

e

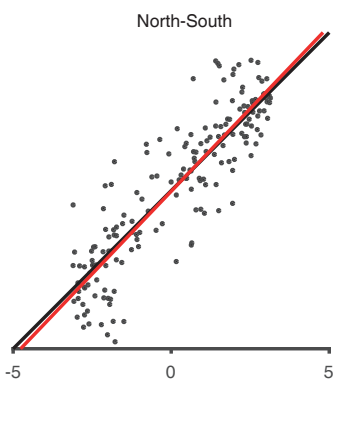

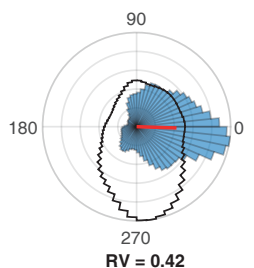

90

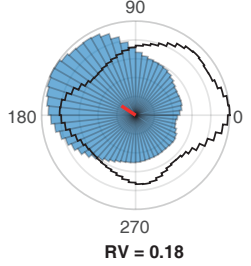

f

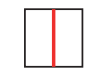

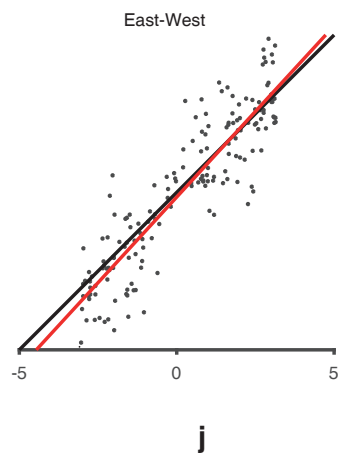

j

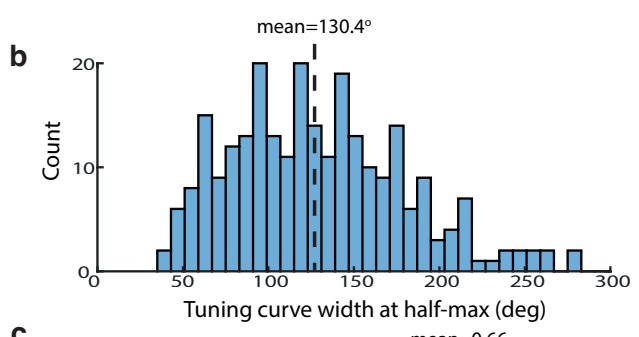

C

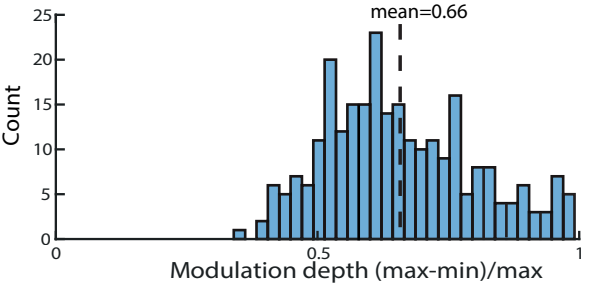

g

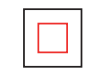

h

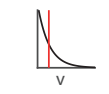

i
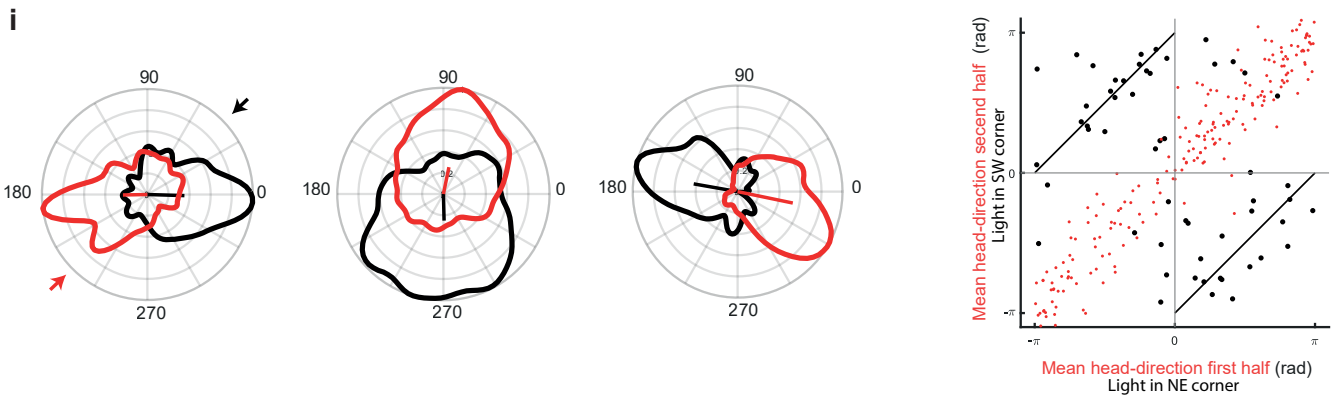

k
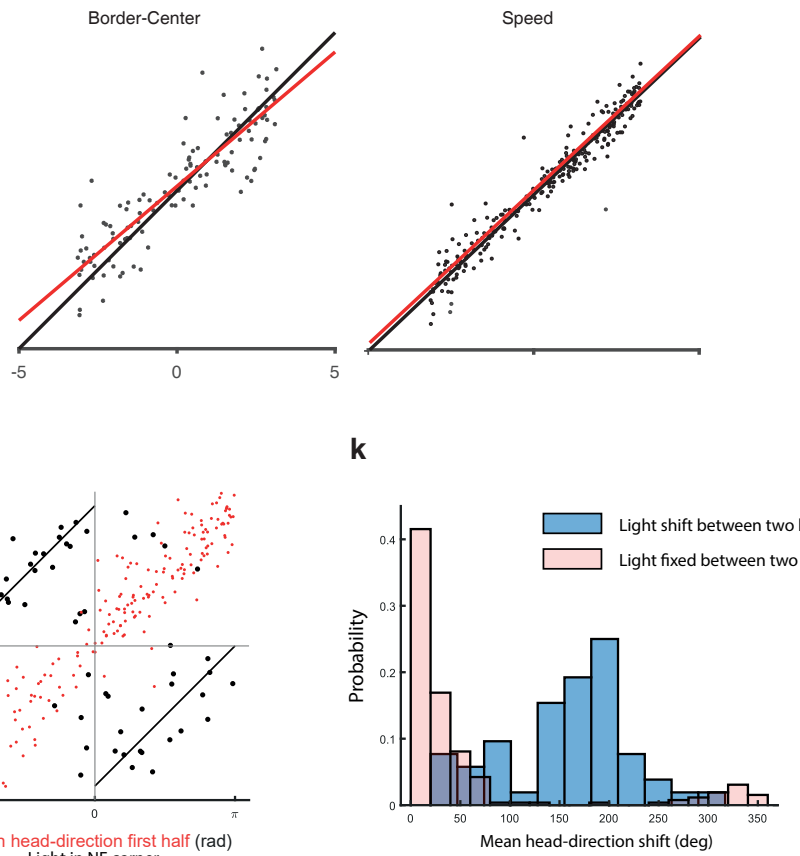

Figure 3. Head-direction cells characteristics, stability, and response to cue-rotation. a, Examples of six cells significantly modulated by head-direction, organized in descending order by their Rayleigh vector (RV; its value is indicated below each neuron). Blue histograms represent the polar firing-rate curve. Black line depicts the time spent in each direction (behavioral curve). Curves are normalized to their maximum. b, Distribution of the tuning curve widths (width at half maximum) of the head direction firing-rate curves. The dashed line represents the population mean. c, Distribution of the modulation depths (maximal firing-rate of the tuning curve minus the minimal firing rate, normalized by the maximal firing rate). The dashed line represents the mean. $\mathbf{d}-\mathbf{h}$, Population scatter plots for HD cells, comparing the preferred head-directions in the first half versus the second half of the session (d), The north versus south parts of the arena (e), east versus west parts of the arena ( $f$ ), near-border versus center of the arena $(\mathrm{g})$, and fast speeds versus slow speeds $(\mathrm{h})$. Black lines are the identity-lines (equal preferred-directions) and red lines show the linear regressions. $\mathbf{i}$, Examples of head-direction tuning curves of three cells before and after $180^{\circ}$ cue rotation (light source switch). Red curves were measured when the light source was in the South-West corner of the arena and black curves when the light source was in the North-East corner (the inset arrows in the left panel designate the directions of the light sources in the arena. Straight lines originating from the center designate the direction and length of the Rayleigh vectors. $\mathbf{j}$, Preferred head-directions in the second half of the session are plotted versus the preferred head-directions in the first half of the session. The black dots show the results when the light source was shifted at mid-session by $180^{\circ}$. For comparison, superimposed in red are the dots from panel $d$ to show the result without a light shift. The black diagonal lines indicate the predicted $180 \mathrm{o}$ shift of the preferred direction. $\mathbf{k}$, Distribution of the differences between preferred head directions measured in the two halves of the session, when the light was shifted by $180 \otimes$ (blue) compared to no shift (red). 

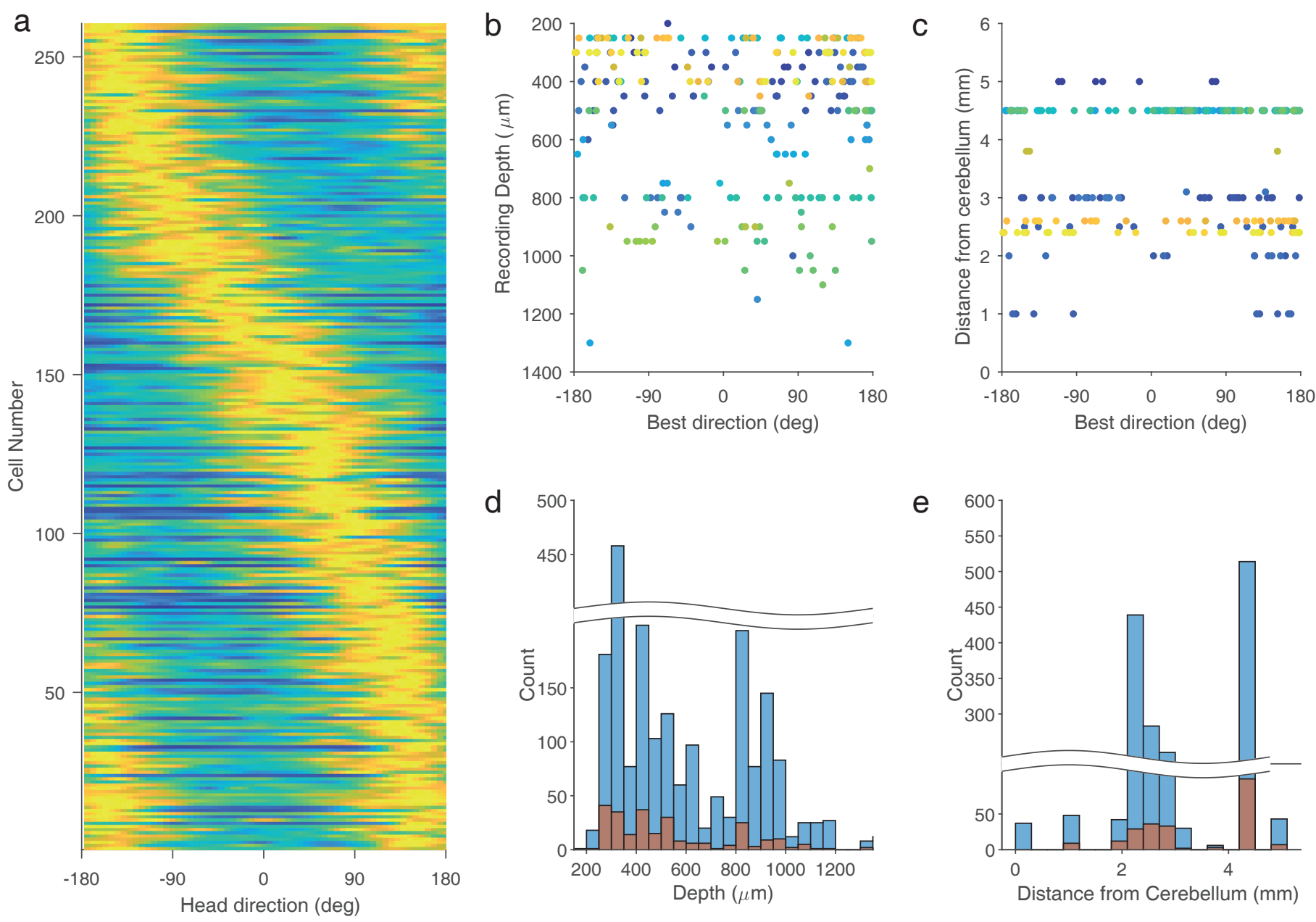

Figure 4. Distributions of preferred directions across the population of head-direction cells. a, Normalized HD tuning-curves for all significant head-direction cells, ordered according to the preferred direction. b, Preferred directions of HD cells as a function of estimated recording depth. Colors designate different quails. c, Preferred directions as a function of recording distance from the cerebellum along the rostro-caudal axis. Colors indicate different quails. d, Histograms of total number of single units recorded (blue) and number of HD cells (orange) as a function of recording depth. e, Same as $d$, but plotted as a function of recording distance from the cerebellum. 